\title{
A NEW METHOD OF MASS MEASUREMENT FOR CHECKWEIGHERS
}

\section{Kengo Fukuda $^{1)}$, Koji Yoshida ${ }^{2)}$, Tetsuya Kinugasa ${ }^{3)}$, Morihito Kamon ${ }^{4)}$, Yoichiro Kagawa ${ }^{5)}$, Toshiro Ono ${ }^{6}$}

1) Oyo Measurement Co., Ltd, Former Manager of Mechanical R\&D Section, 2-12-6, Esaka-cho, Suita, Osaka 564-0063, Japan (k5-fukuda@mvd.biglobe.ne.jp)

2) Okayama University of Science, Department of Mechanical Systems Engineering, 1-1 Ridai-cho, Kita-ku, Okayama-city, Okayama 700-0005, Japan(\k_yoshida@mech.ous.ac.jp, +81862569743)

3) Okayama University of Science, Department of Mechanical Systems Engineering, , 1-1 Ridai-cho, Kita-ku, Okayama-city, Okayama 700-0005, Japan (kinugasa@mech.ous.ac.jp)

4) Yamato Scale Co., Ltd., Department of Automatic Machinery R\&D, 5-22, Saenba-cho, Akashi, Hyogo, 673-8688, Japan (kamon-m@yamato-scale.co.jp)

5) Yamato Scale Co., Ltd., Department of Automatic Machinery R\&D, 5-22, Saenba-cho, Akashi Hyogo, 673-8688, Japan (kagawa-y@yamato-scale.co.jp)

6) Osaka Prefecture University, Professor Emeritus, 1-1 Gakuen-cho, Nakaku, Sakai, Osaka 599-8531, Japan ( t-ono@s9.dion.ne.jp)

\begin{abstract}
A checkweigher is an automatic machine to measure the weight of in-motion products. It is usually located around the end of the production process and ensures the weight of a product within specified limits. Any products are taken out of line if their weights are out of the specified limits. It is usually equipped with an optical device. It is used to make a trigger to set the time duration to allow a product to move completely on the weigh belt for sampling the weight. In this paper, a new method of mass measurement for checkweighers is proposed which uses just signal processing without the optical device. The effectiveness of the method is shown through experiments. Also a possibility of faster estimation of weight is shown.
\end{abstract}

Keywords: checkweigher, weighing method, signal processing, high accuracy.

(C) 2010 Polish Academy of Sciences. All rights reserved

\section{Introduction}

A checkweigher is an automatic machine to measure the weight of in-motion products (i.e., objects). It is usually located around the end of production process and ensures the weight of an object within specified limits. As shown in Fig. 1a), a typical checkweigher consists of three conveyor belts: an infeed belt, a weigh belt, and a reject belt. The weigh belt is usually mounted on a weigh transducer such as a load cell [1]. The weight signal of the transducer is adequately sampled and processed to form a weight. Any objects whose weights are out of the specified limits are taken out of the process line by a sorting device equipped on the reject belt unit. In most cases, an optical device, such as a photo-electric sensor, to detect the passing of an object is mounted between the infeed belt and weigh belt (see Fig. 1b)). The signal from the optical device is used as a trigger to set the time duration to allow the object to move on weigh belt completely for sampling the weight.

If we can make the trigger just by processing the weight signal from the transducer, we would be able to remove the optical device. This leads to bring about a reduction in costs. However, a general signal processing method has not yet been achieved to make the trigger instead of the optical device and to determine the weight of the object, because the checkweigher needs to cope with various types of objects and various conveyor speeds. The difficulty is caused by that checkweighers measure the weights of in-motion objects, hence, 
we have to take weight measurement in the checkweigher as one of "Dynamic mass measurement problem [2-7]".

Ono [2] has worked on a type of checkweighers which has a beam-balance and differential transformer. He has applied modern estimation theory to improve the efficiency of checkweighers in weighing time and accuracy. Then, the feasibility of the proposed method has been shown through simulations and experiments, and also the possibility of dynamic mass measurement has been shown.

Lee et al. [8] have proposed an algorithm to improve the accuracy and weighing time for checkweighers and conveyor belt scales. They have modelled the system as a second order ordinary differential equation. They have proposed to use recursive least square regression method for estimating the parameters in the differential equation when the step input was given. Thereby, mass could be estimated. However, they have only shown the feasibility of the method through simulations.

Kameoka et al. [9] have developed a signal processing method for checkweighers to realize high speed and highly accurate measurement. The checkweighers which they have worked on have a conveyor belt supported by a loadcell at one point and a photo-electric switch. A low pass filter and notch filters are used for smoothing the weight signals from the loadcell. The mass value is calculated as the arithmetic mean of the sampled data of the smoothed weight signal. They have shown through experiments the method is effective. Also, he $[10,11]$ has worked on the effect of floor vibration on the measurement accuracy for checkweighers.

Umemoto et al. $[12,13]$ have worked on checkweighers which have an active weigh belt that is supported on a load cell and photo-electric sensor. The weight signals from the loadcells contain vibrations caused by the conveyor belt. Then, they have proposed to use adaptive notch filters to remove the vibrations in the weight signals. The feasibility has been checked through experiments with a commercially available checkweigher.

As mentioned above, any general signal processing method has not yet been achieved to make the trigger instead of the optical device and to determine the weight of the object. Then, the purpose of this paper is to develop a general algorithm for processing the weight signal to determine the precise moment to begin and finish to sample the weight signal and to determine the accurate weight of an object, that is to say, the accurate mass of the object.

a)

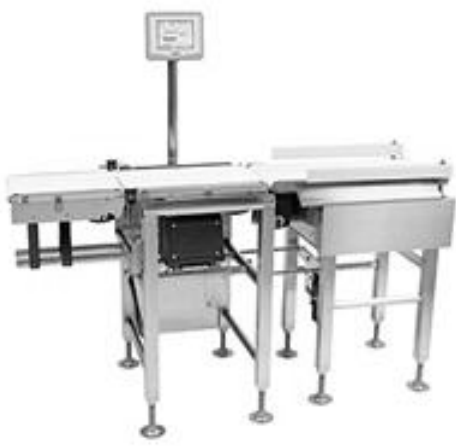

b)

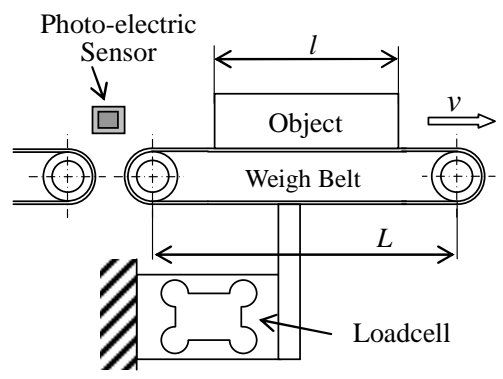

Fig. 1. a) Checkweigher and b) its devices for weighing. 


\section{New method of mass measurement}

\subsection{New method for low speed weighing}

In our researches on Axle Weighing System for In-motion Vehicles [4-7], we have proposed a simple method [7] to determine the part of a weight signal to be processed for weighing based on finding characteristic positions of a differentiated weight signal. This part for weighing is referred to as the effective part in this paper. In the case of axle weighing, the effective part means the segment of the signal when the whole contacting areas of tires fixed on an axle of an in-motion vehicle are on the weighbridge.

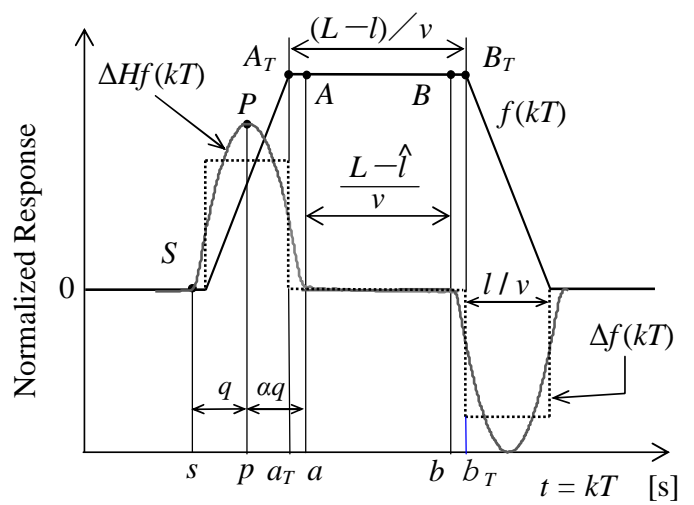

Fig. 2. Signals $f(k T)$ and $\Delta H f(k T)$ contain no vibration components.

In Fig. 2, we show a plot of a weight signal, signals derived from it, and some important points on them indicated with symbols. $f(k T)$ for $k \in Z$ denotes the discrete signal sampled from a weight signal $f(t)$ with sampling time $T$. It should be noted that $f(k T)$ shown in Fig. 2 is a virtual signal which does not contain any vibration component. $\Delta f(k T)$ is a differentiated signal:

$$
\Delta f(k T)=(z-1) f(k T) .
$$

It is smoothed to be a smooth signal:

$$
\Delta H f(k T)=H(z) \Delta f(k T)
$$

by applying a low pass filter $H(z)$. Here, the time delay of $\Delta H f(k t)$ has been corrected.

The weight signals of checkweighers have the same pattern as those of axle weighing systems, then we can apply the proposed simple method to the weight signals of checkweighers. The length of conveyor belt $L$ and conveyor speed $v$ are known when we use a checkweigher. Hence, we can determine the effective time $[a, b]$ of the weight signal by finding the points $S$ and $P$. In Fig. 2, $\alpha$ denotes a positive scalar. Let $l$ denote the length of an object on the conveyor belt as shown in Fig. 1 b). In most cases, we should set $\alpha=1$. If necessary, we can set $\alpha$ appropriately with considering the characteristics of the low pass filter $H(z)$. The obtained weight signal $f(k T)$ from the weight transducer of checkweighers contains vibration components, then we have to use low pass filters. If the conveyor speed is low enough, we can use a higher-order filter which can smooth signals sufficiently. Using 
such filters, we can obtain $\Delta H f(k T)$ sufficiently smooth and apply the simple method as shown in Fig. 2.

However, if the conveyor speed is high, we cannot use such high-order low pass filters. This is because the length of the true effective time:

$$
b_{T}-a_{T}=(L-l) / v
$$

is very short when the conveyor speed becomes very high. The time interval $[s, a]$, in which $\Delta H f(k T)$ is non-zero, increases with the increase in the order of the filter. Hence if we apply the method shown in Fig. 2 to the weight signal $f(k T)$ in this case, we might determine the point $a$ at almost the end of the effective time $b_{T}$ or beyond it. Consequently, the obtained effective time $[a, b]$ would be useless for mass estimation. Then, we have to use lower-order filters. As a result, the point $P$ picked up from $\Delta H f(k T)$, which is not smooth enough, would not be the appropriate point to determine the effective part (see Fig. 5 b)). In this case, we use a new method.

\subsection{New method for high speed weighing}

We take the following steps:

Step 1 Determination of a provisional effective part:

We determine the effective time $[a, b]$ of the weight signal by finding the points $S$ and $P$ in Fig. 2 even though the signal is not smooth enough. Then we take the $A=f(a)$ as the beginning of the effective part provisionally.

Step 2 Design of a FIR filter $G_{k}(z)$ :

By using the least squares method, we design a second order FIR filter whose coefficients are symmetric:

$$
\begin{gathered}
G_{k}(z)=1+\beta_{1}(k) z^{-1}+z^{-2}, \\
\min _{\left.\beta_{1}^{(} k\right)} \sum_{r=k}^{k+n-3} e^{2}(r), \\
e(r)=f((r+2) T)+f(r T)+\beta_{1}(k) f((r+1) T)-C(k)=G_{k}(z) f((r+2) T)-C(k),
\end{gathered}
$$

where $k=a / T, a / T+1, \cdots, b / T-n+1, \quad n \geq 4$.

Step 3 Estimation of the angular frequency of a dominant vibration component:

We calculate zeros $\sigma_{1}(k)$ and $\overline{\sigma_{1}(k)}$ of the filter:

$$
G_{k}(z)=\left(1-\sigma_{1}(k) z^{-1}\right)\left(1-\overline{\sigma_{1}(k)} z^{-1}\right),
$$

where $\overline{\sigma_{1}(k)}$ denotes the complex conjugate of $\sigma_{1}(k)$. Then, we can obtain the dominant angular frequency $\omega_{1}(k T)$ by taking argument of the zero:

$$
\omega_{1}(k T)=\angle \sigma_{1}(k) / T(>0) .
$$

Step 4 Correction of $a$ in Fig. 2:

We pick up a point $k T$ which satisfies the following inequality:

$$
\left(\omega_{0}-\Delta\right) \leq \omega_{1}(k T) \leq\left(\omega_{0}+\Delta\right)
$$


and take it as the correct $a$ which is denoted by $a_{C}$. Where $\omega_{0}$ denotes the dominant angular frequency of the mechanical structure of a weighing system, and $\Delta$ denotes a tolerance value.

In the strict sense, $a_{C}$ is not the point when the object is completely on the weigh belt, however the effect of transient vibration can be neglected.

Step 5 Calculation of mass $m(k T)$ :

By estimating the coefficient in Step 3, we can also obtain an estimated value of the mass $m(k T)$ at $k T$ as follows:

$$
m(k T)=\frac{C(k)}{G_{k}(1) g},
$$

where $g$ is the gravitational constant. Then, $m\left(a_{C}\right)$ would be the earliest mass value estimated with high accuracy.

\section{Experimental Results}

We introduce some results obtained by applying the new method, consisting of Steps mentioned above, to the weight signals $f(k T)$ from the load cell of a checkweigher when an object moved through on the weigh bed with low and high conveyor speeds. These speeds are $30 \mathrm{~m} / \mathrm{min}$ and $80 \mathrm{~m} / \mathrm{min}$, respectively. The checkweigher we used in the experiments is made by YAMATO Scale Co., Ltd. and has an active weigh belt supported on a load cell. Some specifications are tabulated in Table 1.

The objects used for the weighing experiment have the same dimension: $95 \mathrm{~mm}$ x $65 \mathrm{~mm}$ $\times 45 \mathrm{~mm}$, however, the statically measured weight of the object for the low speed weighing is $478.4 \mathrm{~g}$ and that for the high speed weighing is $486.7 \mathrm{~g}$.

In Fig. 3, we show amplitude properties of the filters used for experiments reported in the present paper. Their other characteristics are tabulated in Table 2. We designed two types of low pass FIR filters $H_{L}(z)$ and $H_{S}(z)$ as $H(z)$ of (2) used for smoothing $f(k T)$. Although a band pass filter $H_{B}(z)$ is not used in the new method, it is additionally introduced in Fig. 3. This is because it is used for extracting the dominant vibration component from $f(k T)$ in later discussions.

Table 1. Specifications of the checkweigher.

\begin{tabular}{|l|c|}
\hline \multicolumn{1}{|c|}{ Spec. } & Performance \\
\hline Weighing Range & $6 \sim 600 \mathrm{~g}$ \\
\hline Max Weighing Speed & 330 packs $/ \mathrm{min}$ \\
\hline Sampling Period & $2 \mathrm{~ms}$ \\
\hline Weigh Conveyor Length & $330 \mathrm{~mm}$ \\
\hline
\end{tabular}

Table 2. Descriptions of FIR filters for weight signals.

\begin{tabular}{|l|c|c|c|}
\hline \multicolumn{1}{|c|}{ Notation } & $\mathrm{H}_{\mathrm{L}}(\mathrm{z})$ & $\mathrm{H}_{\mathrm{S}}(\mathrm{z})$ & $\mathrm{H}_{\mathrm{B}}(\mathrm{z})$ \\
\hline \hline Tap number & 101 & 33 & 401 \\
\hline Type & Lowpass & Lowpass & Bandpass \\
\hline Desired cutoff freq. (-6dB) & $5 \mathrm{~Hz}$ & $5 \mathrm{~Hz}$ & $20 \mathrm{~Hz}, 30 \mathrm{~Hz}$ \\
\hline
\end{tabular}




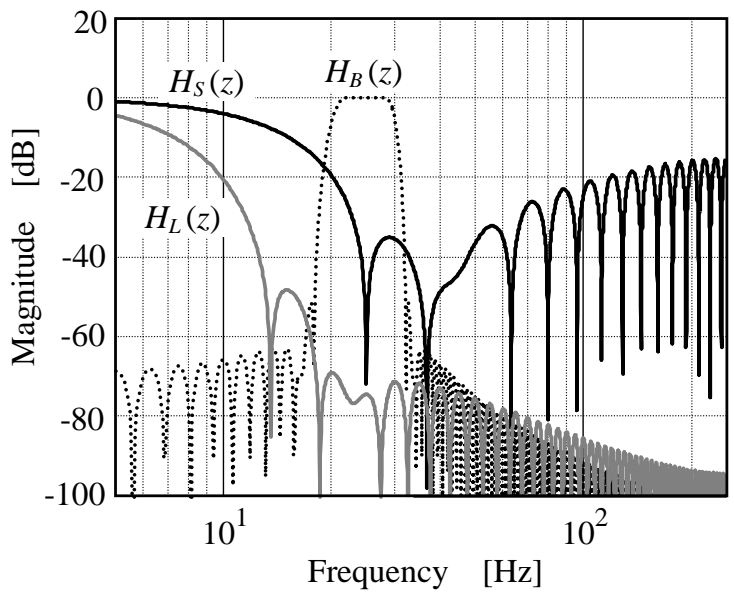

Fig. 3. Amplitude properties of $H_{L}(z), H_{S}(z)$ and $H_{B}(z)$.

\subsection{Example of low speed weighing}

In Fig. 4, we show the weight signal $f(k T)$ in the case that the conveyor speed is $30 \mathrm{~m} / \mathrm{min}$. Its smoothed signal:

$$
H_{L} f(k T)=H_{L}(z) f(k T)
$$

and the smooth differentiated signal $\Delta H_{L} f(k T)$ obtained by applying $H(z)=H_{L}(z)$, whose time delays are corrected, are also shown. Since the data length of the effective part available for measurement is sufficient enough, in this case we can select the higher order filter $H_{L}(z)$ as $H(z)$ in (2). $\Delta H_{L} f(k T)$ processed by a high order filter $H_{L}(z)$ is naturally smooth enough, hence we can apply the simple method shown with Fig. 2. As the result, we have obtained $s=$ $0.978 \mathrm{~s}, p=1.082 \mathrm{~s}$ then:

$$
\begin{aligned}
& q=p-s=0.104 \mathrm{~s}, \\
& a=p+q=1.186 \mathrm{~s}, \\
& \hat{l}=2 q v=0.104 \mathrm{~m},
\end{aligned}
$$

and

$$
b=a+(L-l) / v=s+L / v=0.978+0.309 / 0.5=1.596 \mathrm{~s} .
$$

In this case, since the data length of the effective part is sufficient enough for averaging, we can simply obtain an accurate value by averaging: The average of the filtered weight signal $H_{L} f(k T)$ in the interval $[1.186 \mathrm{~s}, 1.596 \mathrm{~s}$ ] is $478.90 \mathrm{~g}$, which is almost equal to the static value $478.4 \mathrm{~g}$. 


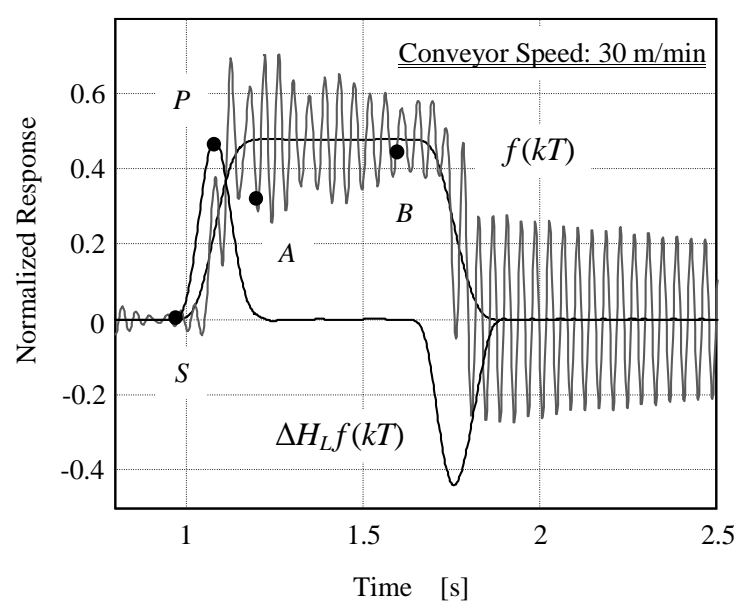

Fig. 4. Example of actual $f(k T), H_{I} f(\mathrm{kT})$ and $\Delta H_{I} f(k T)$ in the case that conveyor speed is $30 \mathrm{~m} / \mathrm{min}$.

\subsection{Example of high speed weighing}

Here, we treat an actual $f(k T)$ obtained in the case that the conveyor speed is $80 \mathrm{~m} / \mathrm{min}$. This is the example of weight signals in high speed weighing that we cannot use a high order filter. In Fig. 5 a), we show $f(k T)$, its smoothed signal $H_{L} f(k T)$ and smooth differentiated signal $\Delta H_{L} f(k T)$ by $H(z)=H_{L}(z)$, in the same manner as in Fig. 4.

As mentioned before, Fig. 5 a) shows a typical example that the determined $a$ is beyond $b$. If we use high order filters such as $H_{L}(z)$, the time delay of the differentiated signal would be so long that we cannot use the simple method illustrated in Fig. 2.

a)

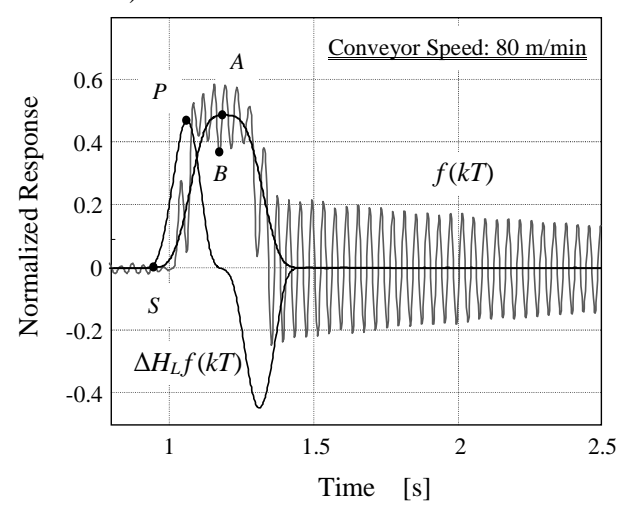

b)

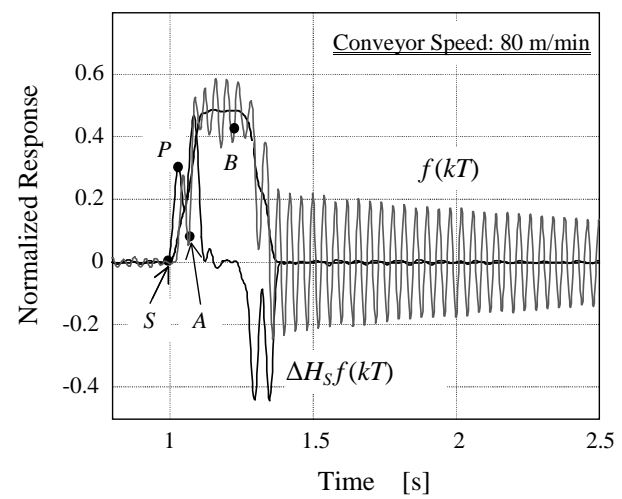

Fig. 5. Example of actual $f(k T)$ and its derivative signals in the case that conveyor speed is $80 \mathrm{~m} / \mathrm{min} ; f(k T), H_{L}$ $f(k T)$ and $\Delta H_{L} f(k T)$ are plotted in a) and $f(k T), H_{S} f(k T)$ and $\Delta H_{S} f(k T)$ are plotted in b).

After all, it might be possible to determine the adequate effective time $[a, b]$ by adjusting coefficient $\alpha$ in Fig. 2, but it is not practical to find effective $\alpha$ for so many kinds of objects to be weighed with using trial-and-error approaches. Although useful $\alpha$ are obtained, we cannot measure weights by averaging. This is because $H_{L} f(k T)$ in $[a, b]$ in Fig. 5a does not contain the flat part as observed in Fig. 4. 
Consequently, we must apply the lower order filter $H_{S}(z)$, whose ability to smooth is not enough, to $f(k T)$. As shown in Fig. $5 \mathrm{~b}, H_{S} f(k T)$ and $\Delta H_{S} f(k T)$ would not be smooth enough. Hence we take Steps of the new method described above.

We can obtain an estimated mass $m(k T)$ and an estimated angular frequency $\omega_{1}(k T)$ at each time $k T$ in Steps 3 and 5 (see Fig. 6), then, we make a pair $\left(\omega_{1}(k T), m(k T)\right)$ at $k T$. In Fig. 7, we show the plots of the pairs; $\omega_{1}(k T)$ is measured along a horizontal axis and $m(k T)$ is measured along a vertical axis, when we set $n=15$. We can see from Fig. 7 that $m(k T)$ is almost constant and close to the static value in the neighbourhood of $\omega_{1}(k T) /(2 \pi)=25 \mathrm{~Hz}$. We can, therefore, tentatively accept $\omega_{0} /(2 \pi)=25 \mathrm{~Hz}$ and $\Delta /(2 \pi)=0.5 \mathrm{~Hz}$. Thus, we would obtain $k T=a_{C}=1.142 \mathrm{~s}$ which is the first moment (earliest time) that satisfies the inequality:

$$
24.5 \mathrm{~Hz} \leq \omega_{1}(k T) /(2 \pi) \leq 25.5 \mathrm{~Hz} .
$$

At the same time, we can obtain $m(1.142)=488.45 \mathrm{~g}$.

a)

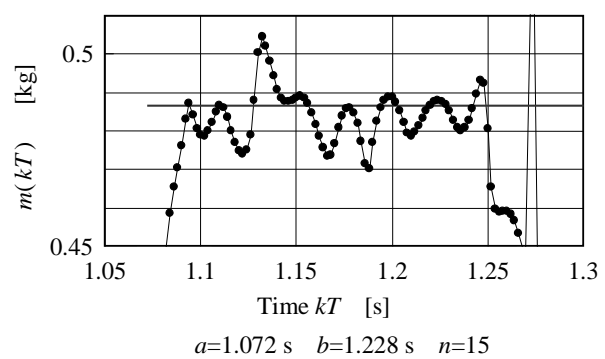

b)

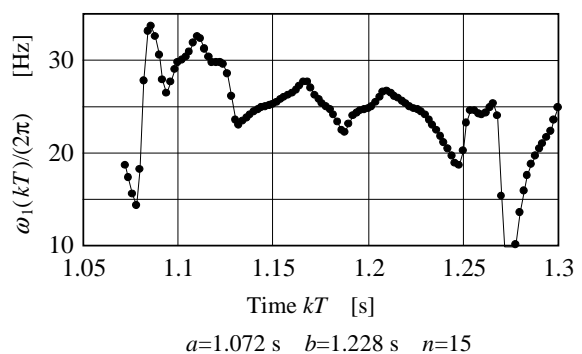

Fig. 6. Estimated values obtained by using $G_{k}(z)$ designed for $f(k T)$ in Fig. 5 ; a) the estimated mass of object $m(k T)$ and b) the estimated angular frequency of dominant component $\omega_{1}(k T) /(2 \pi)$.

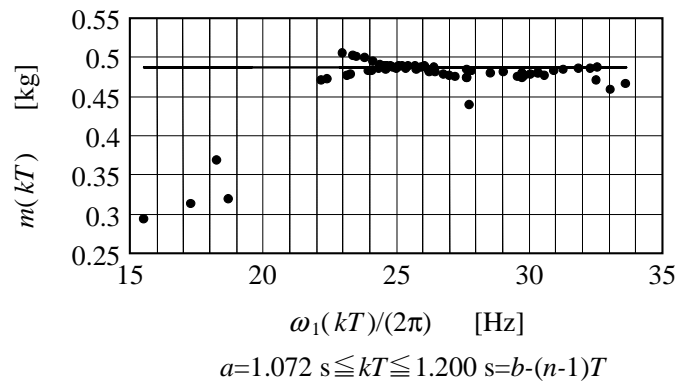

Fig. 7. Plot of estimated angular frequency $\omega_{1}(k T) /(2 \pi)$ and mass $m(k T)$.

If we take an average, a more precise value $\bar{m}$ can be obtained, namely,

$$
\begin{gathered}
\bar{m}=\frac{1}{n_{E}} \sum_{k T \in E} m(k T), \\
E=\left\{k T \mid \omega_{1}(k T) \in\left[\omega_{0}-\Delta, \omega_{0}+\Delta\right], k T \in[a, b-(n-1) T]\right\},
\end{gathered}
$$

where $n_{E}$ is the number of elements in $E$. In this case, $[a, b-(n-1) T]=[1.142 \mathrm{~s}, 1.200 \mathrm{~s}]$, $\Delta /(2 \pi)=0.5 \mathrm{~Hz}$, and $n_{E}=12$, then: 


$$
\bar{m}=487.60 \mathrm{~g} \text {. }
$$

By taking advantage of the observation of Fig. 7, we can also check the estimated mass of the object whether it is accurate or not. For example, the estimated mass at $k T=1.188 \mathrm{~s}$ is $m(1.188)=470.19 \mathrm{~g}$. It would not seem to be accurate, because $\omega_{1}(k T) /(2 \pi)$ at $k T=1.188 \mathrm{~s}$ is estimated to be $22.20 \mathrm{~Hz}$ and

$$
\omega_{1}(1.188) /(2 \pi)=22.20 \mathrm{~Hz} \ll 25 \mathrm{~Hz} .
$$

Actually, $m(1.188)=470.19 \mathrm{~g}$ is far from the static value $486.7 \mathrm{~g}$.

\section{Considerations}

\subsection{Power spectral density of weight signals}

In Fig. 8, we show the estimated power spectral density of the signals truncated by applying a Hanning window to weight signals immediately after objects unload the weigh belt, which is calculated using a 1024 points FFT. The power spectral density of Fig. 8 is very smooth, because it is obtained by taking the average of more than 40 FFTs of sample signals after weighing various kinds of objects against various conveyor speed settings.

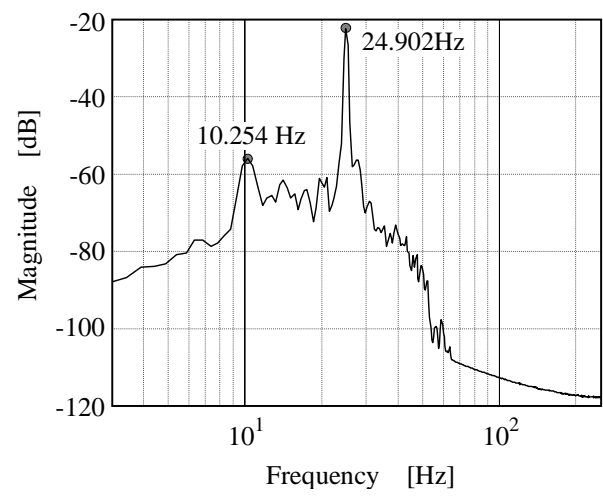

Fig. 8. Estimated power spectral density of weight signal immediately after weighing smoothed by averaging.

We can observe two dominant spectral peaks at $10.3 \mathrm{~Hz}$ and $24.9 \mathrm{~Hz}$. Since the conveyor speeds are not constant, the highest peak at $24.9 \mathrm{~Hz}$ probably indicates the natural vertical vibration due to the weighing structure (the loadcell with the weight belt) and another one at $10.3 \mathrm{~Hz}$, the disturbance due to the floor vibration.

The full scale of the test machine is $600 \mathrm{~g}$ and the static weight of the object is $486.7 \mathrm{~g}$ which was regarded as the true value. Since the spectrum in Fig. 8 obtained when the machine is unloaded, the peak, which indicates the natural frequency, should be shifted to lower during weighing of the object. However, the center frequency of the tolerance range (16), i.e., $25 \mathrm{~Hz}$ that has been determined by observing Fig. 7 is almost equal to the peak frequency in Fig. 8 . This fact suggests that the weighing mechanical structure is not completely expressed as a single-degree-of-freedom mass-spring-damper system. It may be a very important observation in the determination of the tolerance $\Delta$ in the inequality (9). 


\subsection{Effect of disturbance}

The key point of our new weighing method is to design the FIR filter of order two $G_{k}(z)$, which is the most simple structure, on the interval $[k T,(k+n-1) T]$ whose data length is $n$. $G_{k}(z)$ can, therefore, eliminate only one vibration component. For this example, the method is reduced to the design of the most simple filter that notches at the natural frequency around 25 $\mathrm{Hz}$.

Consequently, other vibratory disturbances including the most dominant $10.3 \mathrm{~Hz}$ floor vibration are separated as the residue $e(r)$ through solving the normal equation. In other words, we observe the influence of the $10.3 \mathrm{~Hz}$ vibration component by (16) instead of by evaluating the characteristics of the residue $e(r)$. When $\omega_{1}(k T)$ satisfies $(16), m(k T)$ is adopted as a highly accurate measurement value.

If the magnitudes of disturbances classified as $e(r)$ are relatively large in comparison with that of the natural vibration to be notched, there is a possibility that $\omega_{1}(k T)$ satisfying (16) does not exist, which means that we cannot obtain an aculeate $m(k T)$. Let us observe the magnitudes of the disturbances of the weight signal $f(k T)$ in Fig. 5 by removing the natural vibration with a high order FIR filter.

The upper graph in Fig. 9 shows the output signal.

$$
H_{B} f(k T)=H_{B}(z) f(k T),
$$

where $H_{B}(z)$ is a 401 taps band pass FIR filter whose pass band is [20 Hz, $30 \mathrm{~Hz}$ ] as shown in Fig. 3. The lower one shows the original signal $f(k T)$ (a dashed line) and

$$
\overline{H_{B} f(k T)}=f(k T)-H_{B} f(k T),
$$

which does not contain the $24.9 \mathrm{~Hz}$ component. Measures of the magnitudes of these signals on the effective time interval [1.142 s, $1.228 \mathrm{~s}]$ are tabulated in Table 3. According to the table, if the root mean square of $\overline{H_{B} f(k T)}$ is about one-fifth times as large as that of $H_{B} f(k T)$, the new method is probably successful for high accuracy weighing at high speed.

In any case, we must clarify the relation between the estimated mass $m(k T)$ and dominant angular frequency $\omega_{1}(k T)$ in advance. Even though the disturbance can be neglected, there may exist the cases that we cannot find out the clear relationship like that shown in Fig. 7. Hence, the new method would not be complete from the view point of practical use. We, however, would develop practical techniques taking the advantage of the idea of checking the validity of $m(k T)$ by $\omega_{1}(k T)$ which has been first introduced for checkweighers by the authors.

The weighing method would be seriously affected by a vibratory disturbance whose frequency is smaller than the natural vibration frequency due to the weighing mechanism. We should add the remark that frequencies of disturbances would directly affect the selection rule for the data length $n$ in (5) that is an important parameter in designing the 2nd order FIR filter $G_{k}(z)$ at $t=k T$.

The alternative idea for coping with disturbances is to increase the order of $G_{k}(z)$. That is to say, for example, we replace (4) with:

$$
G_{k}(z)=1+\beta_{1}(k) z^{-1}+\beta_{2}(k) z^{-2}+\beta_{1}(k) z^{-3}+z^{-4},
$$

when we regard the $10.3 \mathrm{~Hz}$ floor vibration as the dominant vibration component to be notched as well as the natural vibration. Nevertheless, at the present time, it would be almost impossible to implement (4)' in commercially available checkweighers, considering the calculation load on its CPU system. Although the order of $G_{k}(z)$ increases, it is also noticed 
that the evaluation of the disturbance is still indispensable for the new method in practical use. At least, we must clarify the condition that guarantees the existence of $\omega_{1}(k T)$ of $G_{k}(z)$ which satisfies (9).

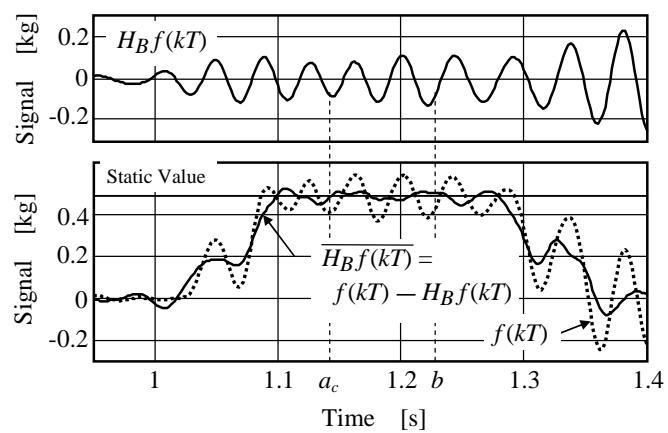

Fig. 9. Dominant vibration $H_{B} f(k T)$ and $\overline{H_{B} f(k T)}=f(k T)-H_{B} f(k T)$.

Table 3. Measures of magnitudes of signals in Fig. 12.

\begin{tabular}{|l|l||l|l|}
\hline & $f(k T)^{* 1)}$ & $H_{B} f(k T)$ & ${\overline{H_{B} f(k T)}}^{* 1)}$ \\
\hline \hline Maximum value & 0.10121 & 0.11706 & 0.02020 \\
\hline Minimum value & -0.11970 & -0.12173 & -0.02067 \\
\hline Peak to peak & 0.22092 & 0.23879 & 0.04087 \\
\hline Root mean square ${ }^{* 2)}$ & 0.07400 & 0.07633 & 0.01490 \\
\hline \hline
\end{tabular}

Unit: $\mathrm{kg}$

*1) Offsets of $f(k T)$ and $\overline{H_{B} f(k T)}$ are cancelled.

*2) Calculated by the rectangular method for numerical integration.

\section{Conclusions}

1. In case that the conveyor speed is lower, we have shown the effectiveness of an algorithm to determine the effective part in weight signals from a checkweigher. The algorithm consists of smoothing the weight signals and finding characteristic points on differentiated weight signals.

2. In case that the conveyor speed is higher, we have proposed a new method which consist of three phases, namely:

a) designing a 2nd order FIR filter whose coefficients are symmetrical;

b) applying it to many local parts of a weight signal in order to estimate the angular frequency of dominant vibration component and the weight in each local part of them;

c) determining the first local part whose estimated frequency is acceptable in comparison with preset value such as the natural frequency of the weighing mechanism. The first point of that part is the beginning point for weighing and the estimated weight in that part would be accurate.

3. The methods mentioned above have been shown effective through experiments.

Consequently, we have shown the possibility to remove the optical device from checkweighers and to achieve a fast weighing method for them.

In the next step, we should confirm whether a microcontroller equipped with an actual checkweigher executes the real time processing of the new method or not. Meanwhile, the evaluation of the weighing accuracy by considering the examples in the present paper is 
insufficient. We wish to clarify the nominal accuracy of the method by the static analysis based on the many results obtained by processing the actual weight signals.

\section{References}

[1] http://www.yamato-scale.co.jp/en/product/detail.php?igpl_no=6\&iprd_no=34

[2] T. Ono: "Applications of Estimation Theory to Dynamic Weighing Problem". Preprints of IMEKO VII

[3] T. Ono, H. Shimaoka: "Dynamic Mass-measurement under Weightless Conditions". Trans. of the Society of Instrument and Control Engineers, vol. 21, no. 11, 1985, pp. 1184-1190. (in Japanese)

[4] K. Fukuda, K. Kameoka, T. Ono, K. Yoshida: "Evaluation of a New Mass-estimation Method for Axle Weights of In-motion Vehicles Using Vehicle Models". Proc. of the 17th International Conference on Force, Mass, Torque and Pressure Measurements, IMEKO TC, 2001, pp. 219-229.

[5] K. Fukuda, H. Tottori, K, Kameoka, T. Ono: “Axle Weighing of In-motion Vehicles”. Trans. of the Society of Instrument and Control Engineers, vol. 38, no. 8, 2002, pp. 653-659. (in Japanese)

[6] K. Fukuda, K. Yoshida, T. Ono, K. Kameoka, T. Hirata: "An Advanced Algorithm for Estimating Axle Weights of In-motion Vehicles". CD-Proc. of the 19th International Conference on Force, Mass \& Torque, IMEKO TC3, 2005.

[7] K. Fukuda, K Yoshida, T. Kinugasa, K. Kanazawa, T. Ono: "A Dynamic Model for the Weighbridge of an Axle Weighing System for In-motion Vehicles with High Velocity". CD-Proc. of APMF, 2007.

[8] W. G. LEE, J. W. Chung and K. P. Kim: "Development of Speed and Accuracy for Mass Measurements in Check Weighers and Conveyor Belt Scales". Proc. of the Second International Symposium on Measurement of Force and Mass between Janan and China, 1994, pp. 23-28.

[9] K. Kameoka, et al.: "Signal processing for checkweigher". Proc. of APMF' 96, 1996, pp. 122-128.

[10] K. Kameoka, H. Sekiguchi: "The Effect of Vibrational Disturbance from a Foundation of a Checkweigher on Weight-checking Accuracy". Trans. of the Japan Society for Precision Engineering, vol. 45, no. 2, 1979, pp. 174-179. (in Japanese)

[11] K. Kameoka, M. Nakatani, G. Komatsu, K. Naito: "Method of the Compensation for Base Vibrations in Mass Measurement - Theory and Its Verification". Trans. of the Society of Instrument and Control Engineers, vol. 35, no. 5, 1999, pp. 616-622. (in Japanese)

[12] Y. Sasamoto, T. Umemoto, M. Adachi, Y. Kagawa: "Frequency Analysis Method using an Adaptive Algorithm with Application to Dynamic Mass Measurement". CD-Proc. of APMF, 2007.

[13]T. Mizui, T. Umemoto, M. Kamon, Y. Kagawa: "Improvement of Accuracy for Continuous Mass Measurement in Cheakweighers with an Adaptive Notch Filter - The Possibility of the Measurement in Steady Time". Proc. of APMF, 2009, pp. 185-191. 